\title{
Palm Fracture Detection using Convolution Neural Network
}

Dr. Harsh S Dave ${ }^{1}$, Dr. Vaishnavi Patel ${ }^{2}$, Dr. Aash Gopalak ${ }^{3}$, Dr. Harsh Bhatt ${ }^{4}$, Dr. Sheshang Degadwala ${ }^{5}$, Dhairya Vyas ${ }^{6}$

1,3,4 MBBS, SBKS MI \& RC, India

\author{
${ }^{2} \mathrm{MD}$, University of Perpetual Help Systems, Phillipines \\ ${ }^{5}$ Associate Professor, Sigma Institute of Engineering, Vadodara, Gujarat, India \\ ${ }^{6}$ Managing Director, Shree Drashti Infotech LLP, Vadodara, Gujarat, India
}

\begin{abstract}
Article Info

Volume 8, Issue 2

Page Number : 594-599

Publication Issue

March-April-2021

\section{Article History}

Accepted : 15 April 2021

Published : 20 April 2021

Palm fractures are due to punching heavy objects (such as a wall or a jaw). A major part of the human body is the palm. Palm provides the hand movement capability. Palm Bone breaks in the human body are fundamental. If the outcome of these fractures is someone's sting in the lips, the skin may be broken. In such instances, wound can be contaminated with bacteria from the other person's mouth and cause illnesses that can permanently impair use of the hand if they are not handled quickly. For analysis of the broken palm, the specialists use the X-ray picture. The manual crack detection is repetitive and the risk of error is high. In order to analyses the broken bone, a robotic system must therefore be developed. In this article, several methods are examined for identification, extraction and characterization. In addition, an inspection is often completed in good conditions and inconvenience. The proposed CNNs are also exploring the expectations of palm bone cracks as positive or negative.

Keywords: X-ray, Median Filter, Morphology Operation, OTSU Transform, $\mathrm{CNN}$
\end{abstract}

\section{INTRODUCTION}

There are many types of bones in the human body. Generally, a car wreck or a horrific fall leads to bone fractures. Due to its vulnerability, the risk of bone breakage is high in mature people. The fractured bone transforms by providing the patient with legitimate therapy. In order to analyse the broken bone, the specialist uses an X-beam or MRI image. The small gap in the bone is difficult for the surgeon to dissect. The manual cycle is repetitive for concluding the broken bone and the probability of blunder is high.
Thus, it is critical to develop a PC dependent structure to reduce the time and error probability for the determination of the crack bone. In clinical imaging, ongoing new AI technologies are commonly used as well as in the hardware sector of force. In the PC-based system, the $\mathrm{x}$-beam or MRI image is used to detect the split bone. The image of the bone has cancer. A correct pre-preparation approximation is then used to remove commotion and borders from the image. And the highlights of the bone image are separated. The structure is finally prepared with highlights, and ML (AI) estimates and deep 
determination are done for the classification how to create CNN which rightly recognises the crack.

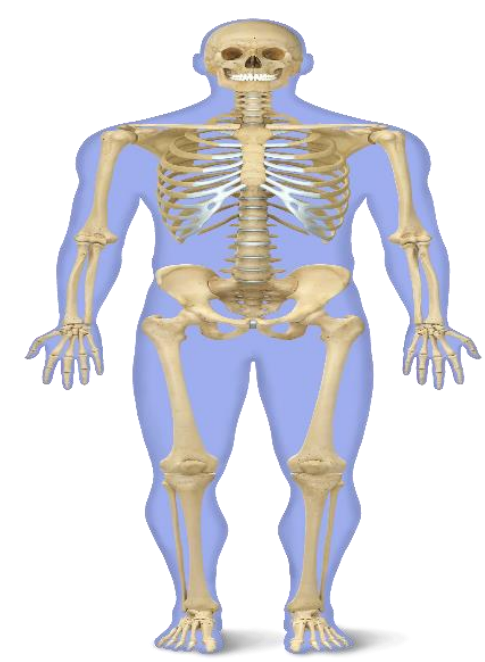

Figure 1: Human Body Bon

In the latter exams, the majority of them discriminate between breaches or intend a general classifier, for instance, manoeuvre through a crack, open break, direct break and switch break. A discreet per trochanteric break classifier is proposed in this article, which identifies five types of trochanteric break. Fig. 1 , the initial image of human body is denoted by the Gaussian channel and lined up, followed by the separation regularisation stage technique to fragment the revenue district (ROI). The identification of the Canny edge is then used to determine the femoral development and then, according to the definition of the tropical crack, the image is insulated into a few bits.

\section{RELATED WORKS}

In [1] The grouping method was developed by deeloping CNN using in-depth learning. Contrasting the sound bone and broken bones data collection provided the frame. The overfitting problem-using keras module has been improved. The last scale of the dataset is 4,000, 2000 of which were sound bones and 2000 were broken bones. The developed CNN with four levels was sufficiently accurate to achieve precision of $92,44 \%$ for bone grouping. On larger information sets the model is still to be attempted. In [2] the developers used a method called "Fuzzy Phrases," which had the option of producing desired results over SVM classification since this approach could individually classify each class and have improved results. The methodology was able to show a vice pinnacle without loss of any data in ex-parcel fluffy sets which can be explored by ascribers. The kimplies were used for the grouping of Fuzzy language and were used for bunching normal and uncommon groups. The best results were achieved with RBF kernels. In this connection. The trials were perframed using 10 overlays and better accuracy and results were obtained.

There have been analyses in [4], 8 of which produced the right difference between tests and proof, 2 of which produced incorrect ID returns. The Contrast includes the line between the neighbouring pixels the distinguishing High estimates of general shifted image tests influence the picture ID in this way rather than various highlights of correlation, energy, and homogeneity. Surface analysis of $\mathrm{x}$-beam images in the lowest possible point of bone breaks uses a GLCM strategy as a technique for retrieval of elements, and $\mathrm{K}$-means as a measure of the clustering results in an accuracy of $80 \%$.

In [5] the decrease in image commotion was finished with one of four GMF, MeF, MF, SSR calculations. The tactic used was to remove the disturbance, by which time the bone line was recognised and the broken bones were distinguished. In order to differentiate the SNAKE'S bone line, the algorithm was used to strengthen the bone line. At that time, external energy was removed to differentiate the split. The results demonstrate that the accuracy achieved was $89 \%$.

In [7] the procedure of regularizations at separation levels is used for ROI portion of the boisterous foundation; the administrator of identification at Canny edge is used to remove breach borders; and afterwards the crack is ordered as a result of a 
trochanteric split in consideration of differences between the reference traditional images and test images. If work is contrasting and exists, unpretentious cracks may be classified by trochanteric. This strategy's exploratory results have become the perfect exhibition.

The Hough Update Guideline [9] applies. The location of the line is completed by a useful adjustment to Hough. The image is examined and separated into one-pixel picture by changing watersheds. The boundary attachment is used to change over a single pixel image into a continuous image. Marker preparation is used at this stage to include an exparcel local number, regional area and various highlights. For dynamic splits, this estimate is not worthwhile.

[10] Multilayer Perceptron and Support Vector Machines have been compiled according to a community of imbalanced learners, who are favourable for modelling the trabecular bone crack zone expectation, using former research methods, Random Under-testing and over-Sampling for Synthetic Minority and classifiers. The optimum mixture (RU+SMOTE) was found to be (MLP) and high-light determination techniques were used to identify biomarkers for the problem of the Crack Zone anticipation.

Deep learning is implemented in the area of break identification and classification in [12]. The organisational model is set up and can accurately predict the kind of broken repositories. The model consists of three layers of convolution and one layer. Via the convolution layer, the enterprise continually removes the highlight data found in its actual limits by inserting this data through an entirely different layer and finally provides the most likely break standard through using the SoftMax work. The neural organisation of the convolution would certainly not overfit, like the conventional neural organisation of BP.

\section{PROPOSED SYSTEM}

As shown in below figure 2 proposed system for Palm Fracture detection are perform in the flow.

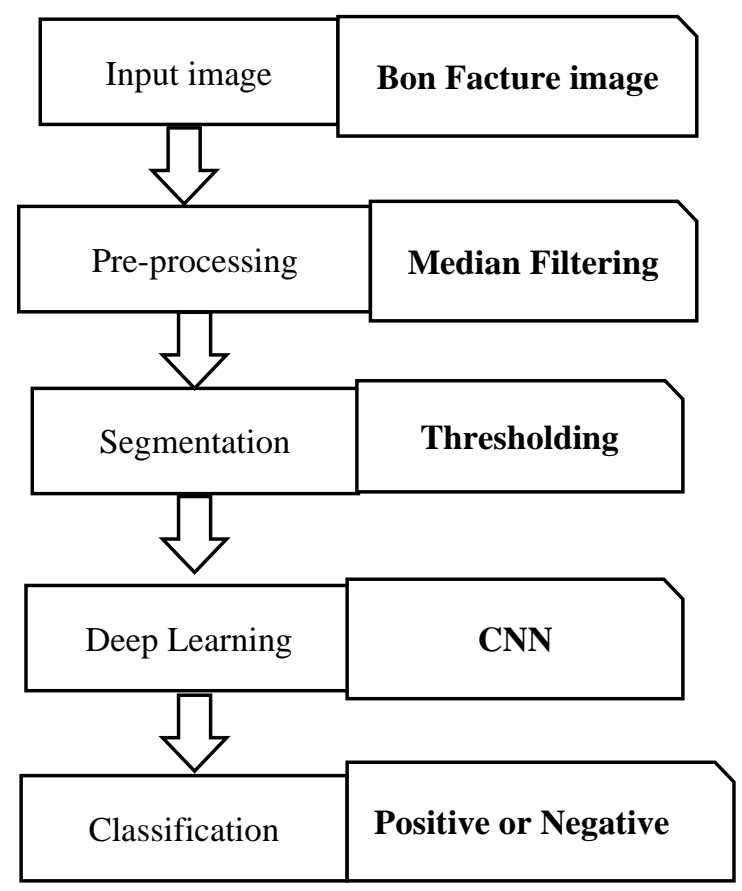

Figure 2: Proposed System Flow Diagram

\section{A. Datasets [1]:}

A large dataset of bone $\mathrm{X}$-beams is MURA (musculoskeletal radiographs). The determination whether the X-Beam study is regular or anomalous is the responsibility for calculations. More than 1.7 billion people worldwide are affected by musculoskeletal conditions which are the most wellknown reasons for severe, long-haul torments and incapacity with an annual increase of 30 million crises visits.

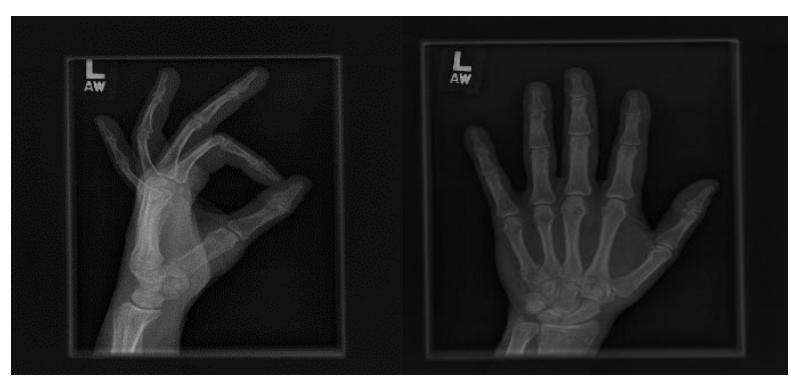

Figure 3: MURA Palm Images 


\section{B. Median Filtering [3]:}

The middle channel is one kind of nonlinear channel. It is exceptionally viable at evacuating drive clamour, the "pepper and salt" commotion, in a picture. The rule of the middle channel is to supplant the dark degree of every pixel by the middle of the dim levels in an area of the pixels, rather than utilizing the normal activity. For middle separating, we indicate the portion size, list the pixel esteems secured by the bit, and decide the middle level.

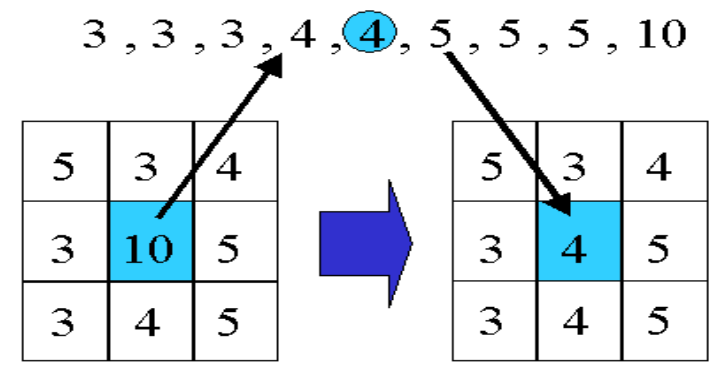

Figure 4: Median Filtering Matrix

\section{Segmentation [6]:}

i.OTUS Thresholding [6]: Otsu method is one of the most successful methods for image thresholding. Converting a greyscale image to monochrome is a common image pro- cessing task. Otsu's method, named after its inventor Nobuyuki Otsu, is one of many binarization algorithms. In the simplest form, the algorithm returns a single intensity threshold that separate pixels into two classes, foreground and background.

ii.Morphological based segmentation [8]: Converting the images to binary reduces computational complexity and storage issues and also is a prerequisite for morphological segmentation of lungs.

- Morphological open operation,

$$
A \circ B=(A \theta B) \oplus B
$$

- Morphological closing operation,

$$
A \bullet B=(A \oplus B) \theta B
$$

\section{Convolution Neural Network}

Convolution Neural Network (CNN) is especially valuable for spatial information examination, picture acknowledgment, PC vision, characteristic language handling, signal preparing, and an assortment of other various purposes. They are organically inspired by the working of neurons in the visual cortex to a visual upgrade. What makes CNN significantly more impressive contrasted with the other criticism forward organizations for picture acknowledgment is the way that they don't need as much human mediation and boundaries as a portion of different organizations, for example, MLP do. This is principally determined by the way that CNN's have neurons organized in three measurements. CNN's make the entirety of this sorcery occur by taking a bunch of info and giving it to at least one of the accompanying fundamental concealed layers in an organization to create a yield.

1. Convolution Layers

2. Pooling Layers

3. Fully Connected Layers

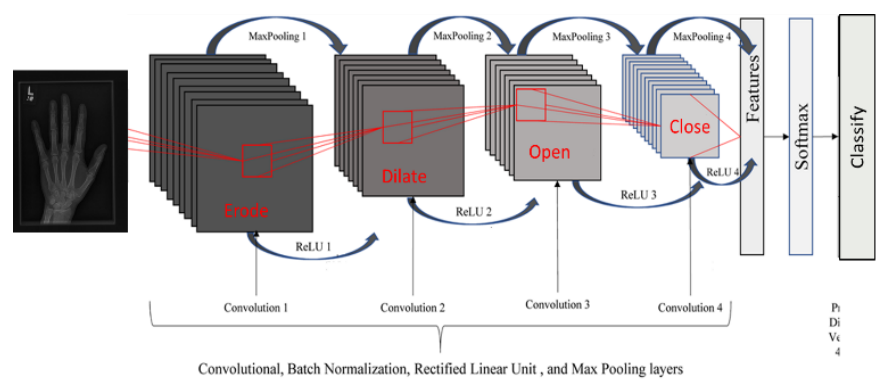

Figure 5: CNN layers

Step 1: Firstly, Take an Input image of Bon Facture

Step 2: By using Prepressing technique Median filter.

Step 3: For Segmentation of image used method Kmean.

Step 4: Using Deep learning Algorithm DNN.

Step 5: After finished above steps finally we have to Classify Facture and its stage which detected by X-ray image.

\section{Simulation Results}

Below Simulation results have been obtained with a Spyder Anaconda. This implementation has shown the robustness, versatility and speed of this smart $\mathrm{x}^{-}$ 
ray device. The findings of cancer identification using the training picture package resulted in $92.94 \%$ Classification.

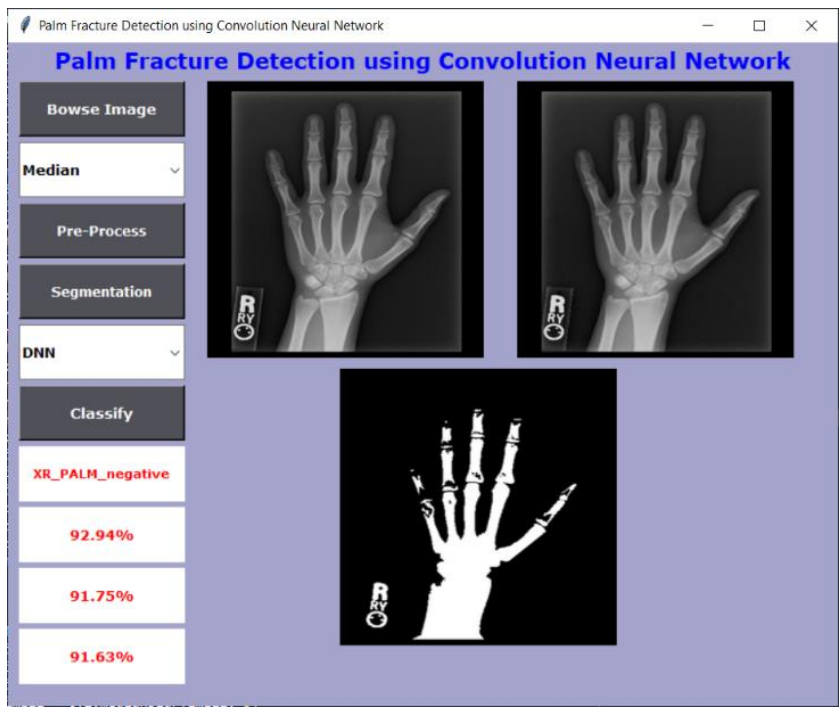

Figure 6: Front Palm

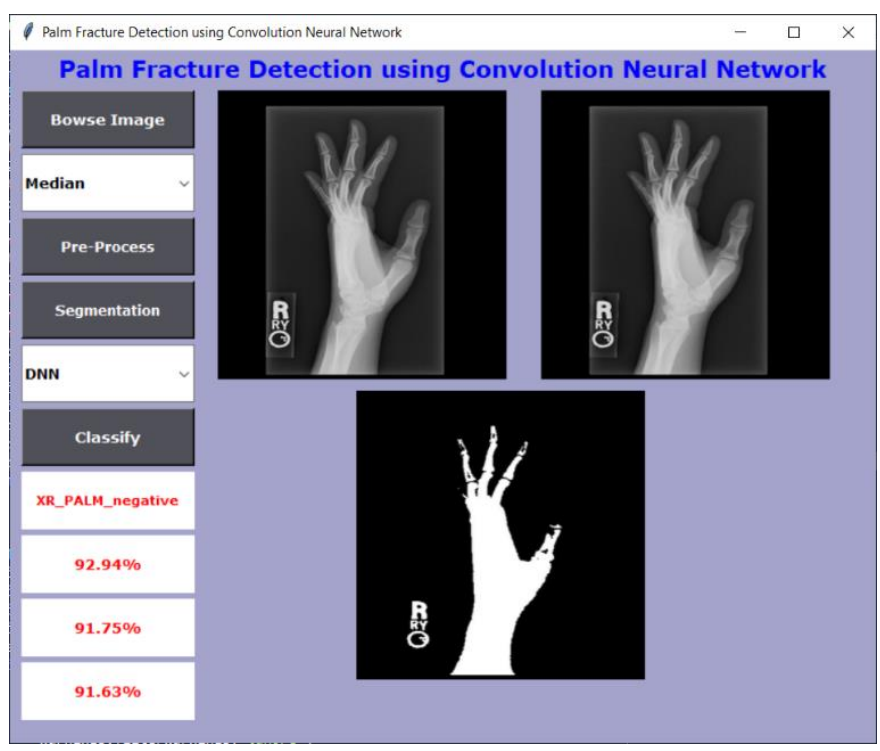

Figure 7: Side Palm

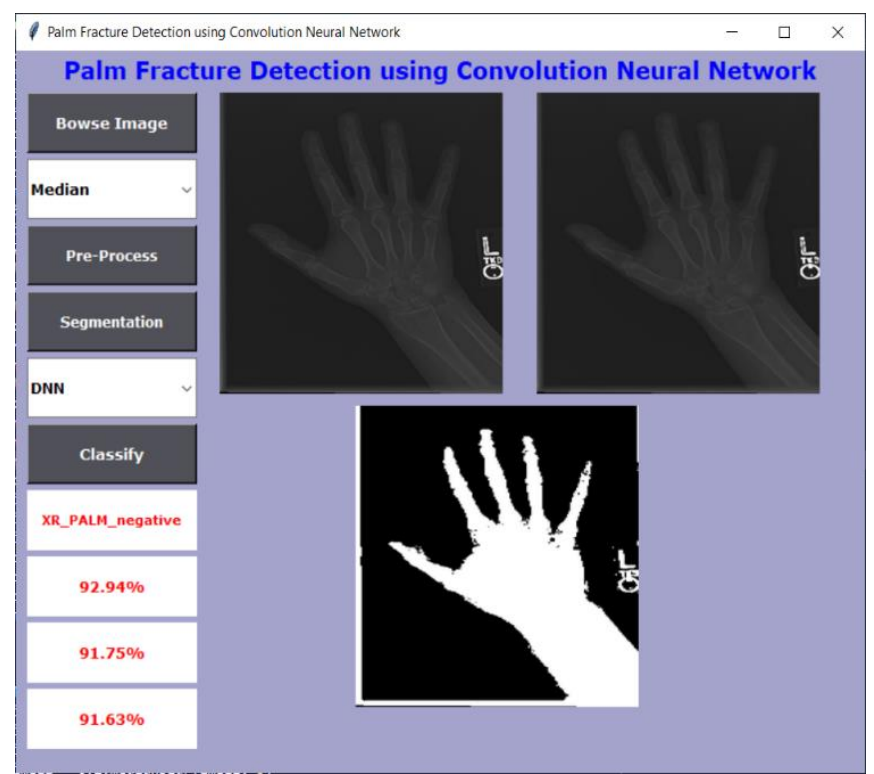

Figure 8: Right Palm

\section{v. CONCLUSION}

Deep learning-based CNN methods for segmentation and classification Palm Fracture have been established in this article. Image processing techniques in medical matters, as in the X-ray machine, are very useful and important. When testing the system, the experimental findings showed that the Palm Fracture screening system produced is a robust image treatment that detects all anomalies in the bon region and particularly in small areas. The stability of the system is to note immediately at the first glance some abnormality in the Palm. In 92.94\% accuracy, the system also directly labels the Positive or Negative in the palm bon, making our system successful.

\section{REFERENCES}

[1] D. P. Yadav and S. Rathor, "Bone Fracture Detection and Classification using Deep Learning Approach," 2020 Int. Conf. Power Electron. IoT Appl. Renew. Energy its Control. PARC 2020, pp. 282-285, 2020, doi: 10.1109/PARC49193.2020.236611.

[2] M. Vasilakakis, V. Iosifidou, P. Fragkaki, and D. Iakovidis, "Bone fracture identification in x-ray 
images using fuzzy wavelet features," Proc. 2019 IEEE 19th Int. Conf. Bioinforma. Bioeng. BIBE 2019, pp. 726-730, 2019, doi: 10.1109/BIBE.2019.00136.

[3] R. Bagaria, S. Wadhwani, and A. K. Wadhwani, "Different techniques for identification of a bone fracture in analysis of medical image," Proc. 2020 IEEE 9th Int. Conf. Commun. Syst. Netw. Technol. CSNT 2020, pp. 327-332, 2020, doi: 10.1109/CSNT48778.2020.9115760.

[4] R. S. Prihatini, A. H. Setyaningrum, and I. M. Shofi, "Texture analysis and fracture identification of lower extremity bones X-ray images," Int. Conf. Electr. Eng. Comput. Sci. Informatics, vol. 2017-December, no. September, pp. 19-21, 2017, doi: 10.1109/EECSI.2017.8239113.

[5] L. Nascimento and M. G. Ruano, "Computeraided bone fracture identification based on ultrasound images," Proc. - 2015 IEEE 4th Port. Meet. Bioeng. ENBENG 2015, no. February, pp. 26-28, 2015, doi: 10.1109/ENBENG.2015.7088892.

[6] Y. Cao, H. Wang, M. Moradi, P. Prasanna, and T. F. Syeda-Mahmood, "Fracture detection in x-ray images through stacked random forests feature fusion," Proc. - Int. Symp. Biomed. Imaging, vol. 2015-July, pp. 801-805, 2015, doi: 10.1109/ISBI.2015.7163993.

[7] L. Wang, H. Cheng, H. Lan, Y. Zheng, and K. Li, "Automatic recognition of pertrochanteric bone fractures in femur using level sets," Proc. Annu. Int. Conf. IEEE Eng. Med. Biol. Soc. EMBS, vol. 2016-October, pp. 3851-3854, 2016, doi: 10.1109/EMBC.2016.7591568.

[8] V. L. F. Lum, W. K. Leow, Y. Chen, T. Sen Howe, and M. A. Png, "Combining classifiers for bone fracture detection in x-ray images," Proc. - Int. Conf. Image Process. ICIP, vol. 1, pp. 1149-1152, 2005, doi: 10.1109/ICIP.2005.1529959.

[9] W. Zheng, N. Ma, H. Sun, and H. Fan, "Feature extraction of $\mathrm{X}$-ray fracture image and fracture classification," 2009 Int. Conf. Artif. Intell. Comput. Intell. AICI 2009, vol. 2, pp. 408-412, 2009, doi: 10.1109/aici.2009.40.

[10] V. C. Korfiatis, S. Tassani, and G. K. Matsopoulos, "A New Ensemble Classification System For Fracture Zone Prediction Using Imbalanced Micro-CT Bone Morphometrical Data," IEEE J. Biomed. Heal. Informatics, vol. 22, no. 4, pp. 1189-1196, 2018, doi: 10.1109/JBHI.2017.2723463.

[11] M. Lotfy, R. M. Shubair, N. Navab, and S. Albarqouni, "Investigation of Focal Loss in Deep Learning Models for Femur Fractures Classification,” 2019 Int. Conf. Electr. Comput. Technol. Appl. ICECTA 2019, pp. 2-5, 2019, doi: 10.1109/ICECTA48151.2019.8959770.

[12] Z. Wu, X. Mo, H. Zhou, L. Liu, and J. Li, "Classification of reservoir fracture development level by convolution neural network algorithm," ICNC-FSKD 2018 - 14th Int. Conf. Nat. Comput. Fuzzy Syst. Knowl. Discov., pp. 243-250, 2018, doi: 10.1109/FSKD.2018.8687232.

\section{Cite this article as :}

Dr. Harsh S Dave, Dr. Vaishnavi Patel, Dr. Aash Gopalak, Dr. Harsh Bhatt, Dr. Sheshang Degadwala, Dhairya Vyas, "Palm Fracture Detection using Convolution Neural Network", International Journal of Scientific Research in Science and Technology (IJSRST), Online ISSN : 2395-602X, Print ISSN : 23956011, Volume 8 Issue 2, pp. 594-599, March-April 2021. Available at

doi : https://doi.org/10.32628/IJSRST2182199 Journal URL : https://ijsrst.com/IJSRST2182199 